'Programa Stricto Sensu en Neurología/Neurociencia de la Universidad Federal Fluminense.

${ }^{2}$ Sector de Trastornos del Movimiento del Hospital Universitario Pedro Ernesto /Neurología/ Universidad del Estado de Río de Janeiro, Brasil.

Recibido el 24 de enero de 2012, aceptado el 2 de septiembre de 2012

Correspondencia a: Giovanna Barros Gonçalves Calle Nelson Gomes de Carvalho, número 130 barrio Vale do Ipê, Juiz de Fora - MG. Cep: 36035410 - Brasil.

E-mail: giovannafst@uol. com.br

\section{Trastorno de la marcha en la enfermedad de Parkinson: freezing y perspectivas actuales}

\author{
GIOVANNA GONÇALVES ${ }^{1,2}$, JOÃO PEREIRA ${ }^{1,2}$
}

\section{Freezing and gait disorders in Parkinson's disease}

More than one third of patients with Parkinson disease experience freezing. It is characterized by the feeling that one's feet are "glued to the floor", and it is more common in the later stages of the disease. The causes of this gait disorder are not yet fully established, but it may lead patients to suffer falls and lose their independence. As a consequence, the development of therapeutic measures which can overcome freezing is of fundamental importance for the autonomy of such individuals. There is no consensus in the literature on the most recommended therapeutic measures for the prevention or attenuation of freezing in gait. What seems to be defined are the phenomenological aspects of the disorder and good therapy, represented by the association between drug therapy and sensorial stimuli or motor coordination training geared towards the specificities to avoid motor difficulties of freezing, when triggering factors are present.

(Rev Med Chile 2013; 141: 758-764).

Key words: Exercise; Freezing; Gait; Parkinson disease; Therapeutics.
$\mathrm{E}$ l congelamiento o "freezing" en la marcha (FM) es un fenómeno caracterizado por episodios transitorios de trastorno motor, mantenido algunos segundos, comúnmente observado en la enfermedad de Parkinson (EP), afectando a más de un tercio de los individuos ${ }^{1-3}$, siendo descrito por los pacientes como la sensación de tener los pies "pegados al suelo" ${ }^{\text {. Puede ser }}$ desencadenado al intentar iniciar y/o continuar la marcha, ante restricciones ambientales que exijan cambios en la velocidad, patrón o sentido del paso con o sin presencia de obstáculos o al caminar en espacios cerrados.

Se observa particularmente en las etapas más avanzadas de la enfermedad y puede ocurrir espontáneamente al andar en espacios abiertos y al aire libre ${ }^{1}$.

A pesar de que el FM presenta mayor frecuencia con la progresión de la enfermedad, Bloem et $\mathrm{a}^{5}$ pusieron en evidencia la presencia de congelamiento en fases precoces, con afectación de hasta $26 \%$ de pacientes con EP no tratados con levodopa (L-dopa). En las fases más avanzadas y con uso prolongado de levodopa, la prevalencia de congelamiento gira en torno de 20 a $60 \%$.

Las causas de FM todavía no son bien comprendidas. Existen varias hipótesis, tales como el congelamiento derivado de la incapacidad de generar una amplitud normal en la longitud del paso $^{6}$, asimetría de la marcha, como evento único, $\mathrm{o}$ incluso problemas en el control motor de esos pacientes. La descoordinación y asimetría de la marcha, pero no aquella relacionada al desempeño motor en general, puede estar asociada al FM ${ }^{1}$.

El FM presenta características distintas de aquellas que ocasionan otros síntomas parkisonianos, no consiguiéndose correlacionar la frecuencia de los episodios de freezing con los síntomas motores de la EP, tales como rigidez y bradicinesia, siendo la frecuencia del FM inversamente proporcional a la presencia del temblor ${ }^{7,8}$. Estos hechos refuerzan las complicaciones para la determinación de la fisiopatología del FM, siendo necesarias más investigaciones para el esclarecimiento de este trastorno ${ }^{7}$. Para completar este análisis, Factor et $\mathrm{al}^{9}$ sugieren que la inestabilidad postural con 
caídas (PIF) y el FM pueden ser subtipos patofisiológicamente distintos de inestabilidad postural o trastornos de la marcha, asociando al PIF y al FM con subscores de marcha mayores y subscores menores de temblor dentro de la clasificación de la Escala Unificada para la Enfermedad de Parkinson. Estos hechos refuerzan la dificultad de determinar la patofisiología del FM, y hacen que se requieran más estudios para aclarar esta afección ${ }^{7}$.

Además de los síntomas comúnmente asociados con la enfermedad de Parkinson, Vandenbossche et al ${ }^{10}$ demuestran que esta afección se relaciona con deficiencias en la resolución de conflictos de la función motora, lo que es importante para comprender la interacción entre los problemas cognitivos y motores. Otras funciones motoras como la resolución de problemas abstractos y la flexibilidad mental no se asocian con el FM.

Los trastornos de la marcha constituyen uno de los síntomas más comunes en la EP, además de ser uno de los principales factores limitantes en la autonomía y la calidad de vida de estos pacientes. La progresión en la enfermedad es caracterizada por déficit en la amplitud y control de la frecuencia de los pasos, provocando la reducción de la amplitud y por consiguiente el aumento de la frecuencia de los pasos ${ }^{11}$. Considerado como un síntoma incapacitante ${ }^{4}$, el FM puede estar asociado a caídas y pérdida de independencia ${ }^{5}$. Por eso, es extremadamente importante el desarrollo de medidas y estrategias terapéuticas que puedan superar el FM, tales como ejercicios que impliquen coordinación, destreza, inicio de la marcha, cambios rápidos de dirección, aumento de la distancia de los pasos, superación obstáculos y movilidad en espacios reducidos ${ }^{12}$.

Esta revisión tiene la finalidad de identificar en la literatura corriente las principales conductas utilizadas para atenuar o prevenir el FM en parkisonianos, destacándose las principales estrategias y trabajos terapéuticos cuya eficiencia y eficacia en el tratamiento de este trastorno motor, hayan proporcionado mejoras en la calidad de vida de esos pacientes.

\section{Desarrollo}

Para esta revisión de literatura se buscó en la base de datos de Medline, Lilacs y Scielo, en el período de 2001 a mayo de 2012, revisiones, relatos de casos, series de casos y estudios experimen- tales, dándose preferencia a artículos originales que investigasen la presencia de congelamiento o "freezing" de la marcha en individuos con EP. Se utilizaron como descriptores en esta investigación: Enfermedad de Parkinson, congelamiento, marcha, ejercicio físico, rehabilitación, fisioterapia y actividad física, en los idiomas portugués, español e inglés. Dos estudios realizados en 1997 y 2000 respectivamente, fueron incluidos por la relevancia de los mismos en esta actualización.

\section{Factores desencadenantes del freezing en la marcha}

Algunos estudios relacionan el FM con la progresión de la enfermedad, refiriendo que la enfermedad en fase avanzada, su gravedad y duración de la terapéutica dopaminérgica facilitan la aparición de este trastorno. Existen evidencias de que la levodopa y los agonistas dopaminérgicos contribuyen al desarrollo del FM, asociados a neurodegeneración y al envejecimiento normal ${ }^{13}$. Otros estudios prospectivos y dobles ciegos relataron mayor frecuencia de FM con el uso de dos antagonistas dopaminérgicos ropinirole ${ }^{14} y$ pramipexole $^{15}$.

El congelamiento que ocurre durante la "fase off" de la EP responde a la levodopa, mientras que los pacientes en "fase on" no presentan esta buena repuesta a la levodopa ${ }^{4}$. Aunque esta condición refuerce la fisiopatología compleja y no definida del FM, la falta de respuesta a la levodopa sugiere posible participación de vías no dopaminérgicas ${ }^{16}$. Otras condiciones también pueden estar implicadas en la génesis de esta afección, así como el FM producido por la incapacidad de generar amplitud normal en la longitud del paso ${ }^{6}$, la marcha descoordinada y asimétrica ${ }^{1}$ y alteraciones en la percepción visual ${ }^{16}$. En la Tabla 1 se encuentran relacionados los factores asociados al desencadenamiento del FM. Por la dificultad e indefinición para establecer los factores causales y la fisiopatología del FM, se vuelve esencial el establecimiento de objetivos y conductas terapéuticas.

\section{Estrategias terapéuticas actuales no medicamentosas para los trastornos de la marcha en la EP}

Entre las intervenciones fisioterápicas para los trastornos de la marcha, deben estar incluidas las terapias convencionales y las terapias con estímu- 
Tabla 1. Factores asociados al desencadenamiento del Freezing en la Marcha

\begin{tabular}{|c|c|}
\hline Autores/año & Factores asociados al desencadenamiento del FM \\
\hline Giladi et al. (2001) & $\begin{array}{l}\text { Evidencias de que la levodopa o agonistas dopaminérgicos puedan contribuir para } \\
\text { el desarrollo del FM }\end{array}$ \\
\hline $\begin{array}{l}\text { Amboni, Barone y Lavarone (2010) } \\
\text { Nicaretta, Pereira y Pimentel (1997) }\end{array}$ & $\begin{array}{l}\text { Pacientes en fase-ON no presentan buena respuesta a la levodopa, sugiriendo } \\
\text { posible participación de vías no dopaminérgicas }\end{array}$ \\
\hline Nieuwboer et al. (2001) & $\begin{array}{l}\text { La incapacidad de generar amplitud normal en la longitud del paso puede des- } \\
\text { encadenar el FM }\end{array}$ \\
\hline Plotnik et al. (2005) & La marcha descoordinada y asimétrica está asociada al FM \\
\hline Nicaretta, Pereira y Pimentel (1997) & $\begin{array}{l}\text { Alteración en la percepción visual puede estar implicada en la génesis de este tipo } \\
\text { de trastorno de la marcha }\end{array}$ \\
\hline
\end{tabular}

los visuales, auditivos y somato-sensitivos ${ }^{6}$. Es de extrema importancia la creación de estrategias de rehabilitación que combinen actividades motoras y estímulos sensoriales ${ }^{11}$, consideradas medidas útiles para superar el congelamiento en la marcha ${ }^{17}$. Se sugiere que la facilitación auditiva rítmica sea un método valioso para la marcha y puede ofrecer, junto al estímulo visual, el resorte necesario para el movimiento secuencial en la $\mathrm{EP}^{18}$.

Nieuwboer et al ${ }^{19}$ verificaron el uso de estímulos sobre todo auditivos, para condicionar ejercicios domiciliares. Los pacientes fueron divididos en dos grupos, el primero recibió tres semanas seguidas de entrenamiento, para después estar tres semanas sin entrenar. El segundo realizó lo contrario y ambos grupos estuvieron las seis semanas siguientes sin entrenarse. Después de varios test, incluyendo cuestionarios de FM, fue constatado que la postura y la marcha mejoraron en $42 \%$ de los casos. La frecuencias de las caídas y de los congelamientos disminuyeron en, respectivamente, $36 \%$ y $5,5 \%$. En las seis semanas sin entrenamiento ambos grupos bajaron en sus metas. Los mismos autores destacaron la reducción en la intensidad del FM, estimando que el entrenamiento con estímulos es mejor que el entrenamiento sin la existencia de estímulos.

La utilización de estímulos auditivos es considerada fundamental para mejorar los movimientos, aumentando también la cadencia y la longitud del paso en individuos con EP. Aunque se hayan obtenido efectos positivos en el uso de pistas sonoras como estrategias para mejorar la marcha, la facilitación auditiva no siempre mejora la variabilidad y los distintos aspectos de la marcha de estos individuos ${ }^{18}$.
Pacientes con EP pueden generar patrones adecuados de marcha en presencia de estimulación sensorial regular. Estímulos visuales pueden normalizar la longitud del paso de varios minutos a varias horas. La utilización de pistas visuales con tiras blancas colocadas en el suelo pueden ser útiles para señalizar el camino y guiar a los individuos entre las estancias del domicilio. Estas pequeñas señales pueden ser colocadas también en paredes y puertas, donde episodios de congelamiento ocurren con frecuencia, para recordar a estos pacientes que adopten medidas de precaución durante los desplazamientos ${ }^{20,21}$.

Uno de los métodos más comunes de estímulo visual es el uso de pistas colocadas en el suelo y líneas terrestres ${ }^{17}$ para auxiliar el inicio y la ejecución de la marcha ${ }^{18}$. La utilización de luces de las más diversas formas puede ser de gran utilidad. Estudios de Van Wegen et $\mathrm{al}^{22}$ verificaron que la utilización de luces parpadeando rítmicamente, así como proyecciones virtuales de rayas en el suelo podrían mejorar la longitud de los pasos en la EP. Otros dos estudios contradicen lo mismo, así, Lebold y Almeida ${ }^{17}$ al utilizar pistas visuales por dispositivo láser (flujo óptico) y Kompoliti et $\mathrm{a}^{23}$ empleando un dispositivo de señalización visual de haz de láser proyectado a partir de la extremidad distal de una bengala, no consiguieron la mejora, así como la reducción del número de episodios de FM.

En contrapartida, se destaca como resultado positivo la hipótesis que relaciona el aumento de la atención como responsable de la mejoría en la longitud del paso en individuos con $\mathrm{EP}^{17}$. Anteriormente Olmo y Cudeiro ${ }^{18}$ ya sugerían el uso de estrategias de atención, como ensayar mental- 
Freezing en enfermedad de Parkinson - G. Gonçalves et al

Tabla 2. Estrategias terapéuticas actuales para los trastornos de la marcha que presentan los parkinsonianos

\begin{tabular}{|c|c|c|}
\hline Autores/año & Estrategias terapéuticas & $\begin{array}{l}\text { Resultados sobre los trastornos de la } \\
\text { marcha }\end{array}$ \\
\hline $\begin{array}{l}\text { Nieuwboer et al. } \\
\text { (2007) }\end{array}$ & $\begin{array}{l}\text { Uso de estímulos auditivos para condicionar } \\
\text { ejercicios domiciliares }\end{array}$ & $\begin{array}{l}\text { I. Mejora de la postura y de la marcha en un } 42 \% \\
\text { II. Reducción de las caídas (36\%) y de los congela- } \\
\text { mientos }(5,5 \%)\end{array}$ \\
\hline $\begin{array}{l}\text { Olmo y Cudeiro } \\
(2005)\end{array}$ & $\begin{array}{l}\text { Pistas sonoras como estrategias para mejorar la } \\
\text { marcha }\end{array}$ & $\begin{array}{l}\text { Mejora de los movimientos, aumentando la caden- } \\
\text { cia y la longitud de la zancada en individuos con EP }\end{array}$ \\
\hline $\begin{array}{l}\text { Aminian et al. } \\
\text { (2002) y Salarian } \\
\text { et al. (2004). }\end{array}$ & $\begin{array}{l}\text { Pistas visuales a través de tiras blancas colocadas } \\
\text { en el suelo para señalizar el camino que guíe a } \\
\text { los individuos entre las estancias del domicilio }\end{array}$ & $\begin{array}{l}\text { Estímulos visuales pueden normalizar la longitud del } \\
\text { paso durante varios minutos a varias horas }\end{array}$ \\
\hline $\begin{array}{l}\text { Van Wegen et al. } \\
(2006)\end{array}$ & $\begin{array}{l}\text { Luces parpadeando rítmicamente y proyecciones } \\
\text { virtuales de rayas en el suelo }\end{array}$ & $\begin{array}{l}\text { Usados para mejorar la longitud de los pasos en } \\
\text { personas con EP }\end{array}$ \\
\hline $\begin{array}{l}\text { Lebold \& Almeida } \\
(2010)\end{array}$ & $\begin{array}{l}\text { Pistas visuales por dispositivo de láser (flujo } \\
\text { óptico) }\end{array}$ & Puede no tener éxito en la mejora del FM \\
\hline $\begin{array}{l}\text { Kompoliti y cols. } \\
\text { (2000) }\end{array}$ & $\begin{array}{l}\text { Dispositivo de señalización visual de haz de láser } \\
\text { proyectado a partir de la extremidad distal de } \\
\text { una bengala }\end{array}$ & No redujo el número de episodios de FM \\
\hline $\begin{array}{l}\text { Olmo y Cudeiro } \\
(2005)\end{array}$ & $\begin{array}{l}\text { Uso de estrategias de atención, como ensayar } \\
\text { mentalmente el próximo movimiento durante } \\
\text { la ejecución }\end{array}$ & Mejora el patrón de marcha de parkinsonianos \\
\hline $\begin{array}{l}\text { Rochester et al. } \\
\text { (2005) }\end{array}$ & $\begin{array}{l}\text { Señales auditivas promovidas por ritmos y soni- } \\
\text { dos musicales }\end{array}$ & Auxiliar y facilitar la locomoción \\
\hline
\end{tabular}

mente el próximo movimiento a ejecutar y evitar distracciones durante la tarea, como conductas eficientes para mejorar el patrón de marcha en la EP. Estas observaciones llevaron a intervenciones con diferentes estrategias fisioterapéuticas para mejorar la marcha de parkisonianos, tales como estrategias de atención y utilización de señales auditivas rítmicas, como músicas. Rochester et $\mathrm{al}^{24}$ destacan las señales auditivas promovidas por ritmos y sonidos musicales, que pueden auxiliar y facilitar la locomoción.

Las estrategias terapéuticas actuales para los trastornos de la marcha en la EP pueden ser mejor visualizadas en la Tabla 2.

A pesar de estas constataciones, los mecanismos neurofisiológicos subyacentes a esa capacitación sensorial todavía tienen que ser determinados ${ }^{11}$, así como las investigaciones que emplean pistas visuales y señales auditivas en la prevención de FM. De hecho tienen importancia las informaciones visuales y auditivas, no siendo, sin embargo, los únicos factores que contribuyen en la mejoría en el FM.

\section{Procedimientos actuales para el tratamiento del FM}

La realización de actividades que exijan agilidad sensitivomotora ha sido destacada en la literatura de divulgación de programas de rehabilitación específicos aplicados en los diversos trastornos de la EP. Se destaca en el FM ejercicios que envuelven coordinación, destreza, inicio de la marcha, mudanzas rápidas en la dirección y sentido, tamaño de los pasos y zancada superando obstáculos y la movilidad en espacios reducidos. Con la superación de estas actividades hay exigencias de nuevos ejercicios para realizar estas tareas con mayor velocidad y de forma más compleja, con asociaciones a tareas cognitivas dobles y utilización de espacios más estrechos ${ }^{12}$.

Estudios recientes pusieron en evidencia nuevas perspectivas en las actividades motoras a ser utilizadas en el tratamiento no medicamentoso de parkisonianos con FM. Entre estos se destacan el estudio piloto de Lo et $\mathrm{a}^{25}$, donde parkisonianos en entrenamiento de la marcha asistida en una 
estera con sistema de soporte de peso corporal, con utilización de órtesis de marcha automática (Lokomat), después de diez sesiones de $30 \mathrm{~min}$, presentan reducción de FM, con mejoras también en la velocidad de la marcha, longitud del paso, ritmicidad y coordinación.

Otro estudio, realizado por Snijders y Bloem ${ }^{26}$ evidenció la capacidad de los parkisonianos con FM de montar en bicicleta, a pesar de que el congelamiento en la marcha no desaparece y retorna después de terminar esta actividad. Especulando el resultado, se puede suponer que la rotación de los pedales de la bicicleta puede actuar como estímulo externo, permitiendo que el ciclismo, con los movimientos continuos, ofrezca un enfoque útil y agradable a los pacientes con FM.

En el tratamiento con medicamentos, Espay et $\mathrm{al}^{27}$ analizaron el uso de metilfenidato (MPD) en la marcha de diecisiete parkinsonianos divididos al azar en un grupo experimental con MPD (80 $\mathrm{mg} /$ día) y un grupo de control (placebo) durante 12 sem de tratamiento. Luego del experimento, los pacientes que habían recibido una evaluación del largo del paso y la velocidad de la marcha, con la Escala Unificada para la Enfermedad de Parkinson (UPDRS) y el cuestionario de freezing en la marcha (FOGQ) y que también habían recibido mediciones de depresión, somnolencia y calidad de vida mostraron mejoras en algunas medidas de depresión; mientras que las variables de la marcha no mostraron cambios y los resultados de la UPDRS y del FOGQ empeoraron en el grupo experimental con metilfenidato en comparación con el grupo placebo, sí hay mejoras en las medidas de función motora, somnolencia y calidad de vida.

Entre los procedimientos quirúrgicos para el tratamiento de FM, está la estimulación cerebral profunda en el núcleo pedúnculo pontino. Otras respuestas distintas fueron observadas en el estudio de tres pacientes con EP avanzada y congelamiento incapacitante y resistente a la levodopa. El primer paciente presentó mejora acentuada en el FM entre 2 y 6 sem después de la cirugía, mientras que el segundo tuvo mejora transitoria. El tercer paciente no presentó mejora perceptible de este trastorno. Aunque con resultados preliminares, este estudio muestra la necesidad de más investigaciones para el ajuste en los parámetros de estimulación y optimización para mejora del congelamiento. A pesar de la respuesta satisfactoria al procedimiento quirúrgico, la mejora del FM no fue mantenida en ninguno de los tres pacientes ${ }^{28}$.

Volviendo al uso del método de simulación en el PPN, Thevathasan et $\mathrm{al}^{29}$ analizaron tres grupos: 8 parkinsonianos con FM grave, 8 pacientes con Parkinson sin fibromialgia y 10 sujetos sanos agrupados por edad. En los pacientes parkinsonianos con fibromialgia y en los individuos sanos pudo observarse el tiempo de reacción causado por un estímulo auditivo fuerte. Sin embargo, esta reacción no se presentó en los pacientes parkinsonianos con FM severa.

Moreau et a ${ }^{30}$ compararon los efectos de varios parámetros de estimulación cerebral profunda del núcleo subtalámico (STN-DBS) en el FM, a fin de determinar si tales efectos están relacionados con la energía o frecuencia de estimulación. Para eso fueron evaluados los parámetros STN-DBS de 13 parkisonianos y comparados los efectos sobre la marcha de dos tensiones diferentes: tensión habitual del paciente [Mediana 3 voltios] y alta tensión [Mediana 3,7 voltios] y dos frecuencias diferentes ( 60 y $130 \mathrm{~Hz}$, manteniendo la misma energía total proporcionada) versus "off-estimulación". Como resultado, el número de episodios de congelamiento fue significativamente menor en la tensión de 60 $\mathrm{Hz}$ y mayor en la tensión de $130 \mathrm{~Hz}$ (a pesar de no alcanzar significancia estadística) de que para "offestimulación”. Así, se sugieren nuevas estrategias para etapas diferentes de sucesos, con STN-DBS a $130 \mathrm{~Hz}$ y tensión habitual durante los primeros años de la STN-DBS y $60 \mathrm{~Hz}$ en alta tensión en parkisonianos que desarrollaran trastornos graves en la marcha. En resumen, los procedimientos actuales para el tratamiento del FM pueden ser visualizados en la Tabla 3.

El empleo de distintos métodos para el tratamiento del FM como medicamentos, cirugía, estímulos visuales y auditivos, estrategias de mejora de la atención y entrenamientos motores específicos, así como la combinación de estas medidas terapéuticas, parecen ser las estrategias más útiles para superar y prevenir el FM, con posibilidad de aumentar la independencia y autonomía de estos individuos.

\section{Conclusiones}

En esta revisión se puede verificar que no existe un consenso en la literatura entre las medidas terapéuticas más indicadas para prevenir o atenuar 
Freezing en enfermedad de Parkinson - G. Gonçalves et al

Tabla 3. Procedimientos recientes para el tratamiento del Freezing en la marcha

\begin{tabular}{|c|c|c|}
\hline Autores/año & Perspectivas actuales & Objetivos terapéuticos \\
\hline $\begin{array}{l}\text { King y Horak } \\
\text { (2009) }\end{array}$ & $\begin{array}{l}\text { I. Ejercicios que implican coordinación, destreza, inicia- } \\
\text { ción de la marcha, mudanzas rápidas de dirección, } \\
\text { pasos laterales superando obstáculos y movilidad en } \\
\text { espacios reducidos para o FM } \\
\text { II. La progresión de los ejercicios exige realización } \\
\text { de estas tareas y mayor velocidad y de forma más } \\
\text { compleja, asociando tareas cognitivas dobles (senso- } \\
\text { motoras) y en espacios más estrechos }\end{array}$ & Mejora del Freezing en la marcha \\
\hline $\begin{array}{l}\text { Albert et al. } \\
(2010)\end{array}$ & $\begin{array}{l}\text { I. Actividad: Entrenamiento de marcha asistida en una } \\
\text { estera con sistema de soporte de peso corporal } \\
\text { II. Diez sesiones de } 30 \mathrm{~min}\end{array}$ & $\begin{array}{l}\text { Reducción del FM y mejora en la velocidad, } \\
\text { longitud de la zancada, ritmicidad y coordi- } \\
\text { nación }\end{array}$ \\
\hline $\begin{array}{l}\text { Snijders y Bloem } \\
(2010)\end{array}$ & $\begin{array}{l}\text { Parkinsonianos con FM poseen capacidad preservada } \\
\text { para montar en bicicleta, sin embargo el congelamiento } \\
\text { de la marcha retorna después de terminar la actividad }\end{array}$ & $\begin{array}{l}\text { Permitir que el ciclismo ofrezca un enfoque útil } \\
\text { y agradable a los pacientes con FM }\end{array}$ \\
\hline $\begin{array}{l}\text { Muhammad, } \\
\text { Nashatizadeh y } \\
\text { Jankovic (2008) }\end{array}$ & $\begin{array}{l}\text { Fue realizada la estimulación cerebral profunda del } \\
\text { núcleo pedúnculo-pontino en tres pacientes con EP } \\
\text { avanzada e incapacitante, congelamiento resistente a } \\
\text { la levodopa }\end{array}$ & $\begin{array}{l}\text { Mejora del FM en dos de los tres pacientes } \\
\text { estudiados. Sin embargo, el mantenimiento } \\
\text { de los resultados positivos no fue observado } \\
\text { en ninguno de los tres pacientes }\end{array}$ \\
\hline $\begin{array}{l}\text { Moreau et al. } \\
(2008)\end{array}$ & $\begin{array}{l}\text { La investigación analizó STN-DBS no FM, buscando } \\
\text { determinar si tales efectos están más relacionados a la } \\
\text { energía o frecuencia de estimulación }\end{array}$ & $\begin{array}{l}\text { Estrategias para estados de la enfermedad, con } \\
\text { STN-DBS a } 130 \mathrm{~Hz} \text { y tensión habitual durante } \\
\text { los primeros años y } 60 \mathrm{~Hz} \text { en alta-tensión en } \\
\text { trastornos graves da marcha }\end{array}$ \\
\hline
\end{tabular}

el congelamiento o freezing en la marcha. Lo que parece definido son los aspectos fenomenológicos del trastorno, a pesar de que esta deducción derive en la mayoría de los artículos de estudios de caso o revisiones. Por el momento se puede destacar como buena terapia la asociación entre el tratamiento medicamentoso y los estímulos sensoriales y/o entrenamientos motores enfocados a las especificidades y dificultades motoras del FM presentadas por estos individuos, en la presencia de factores desencadenantes del congelamiento. Estrategias que implican la iniciación y progresión de la marcha, el tamaño de los pasos con la superación de obstáculos, mudanzas rápidas de dirección y sentido, movilidad en espacios reducidos y superación de situaciones estresantes, podrán modificar los aspectos sintomáticos derivados de este trastorno, siendo de gran utilidad práctica.

De esta forma, se deben continuar los estudios para determinar la terapéutica más indicada para la superación, atenuación y prevención de este trastorno de la marcha, no olvidando que se trata de una enfermedad crónica y neurodegenerativa de evolución progresiva del sistema nervioso central.

\section{Referencias}

1. Plotnik M, Giladi N, Balash Y, Peretz C, Hausdorff JM. Is Freezing of Gait in Parkinson's Disease Related to Asymmetric Motor Function? American Neurological Association 2005; 57: 656-63.

2. Moore O, Peretz C, Giladi N. Freezing of Gait Affects Quality of Life of Peoples with Parkinson's Disease Beyond Its Relationships with Mobility and Gait. Movement Disorders 2007; 22: 2192-5.

3. Chee R, Murphy A, Danoudis M, Georgiou-karistianis N, Iansek R. Gait freezing in Parkinson's disease and the stride length sequence effect interaction. Brain a journal of neurology 2009; 132: 2151-60.

4. Amboni M, Barone P, Iavarone A. A Two-Year FollowUp Study of Executive Dysfunctions in Parkinsonian Patients with Freezing of Gait at On-State. Movement Disorders 2010; 25: 798-804.

5. Bloem BR, Hausdorff JM, Visser JE, Giladi N. Falls and Freezing of Gait in Parkinson's Disease: A Review of Two Interconnected, Episodic Phenomena. Movement Disorders 2004; 19: 871-84.

6. Nieuwboer A, Dom R, De Weerdt W, Desloovere K, Fieuws S, Broens-kaucsik E. Abnormalities of the spatiotemporal characteristics of gait at the onset of freezing 
in Parkinson's disease. Movement Disorders 2001; 16: 1066-75.

7. Bartels AL, Balash Y, Gurevich T, Schaafsma JD, Hausdorff JM, Giladi N. Relationship between freezing of gait (FOG) and other features of Parkinson's: FOG is not correlated with bradykinesia. Journal of Clinical Neuroscience 2003; 10: 584-8.

8. Giladi N. Freezing of gait. Advances in Neurology 2001; 87: 191-7.

9. Factor SA, Steenland NK, Higgins DS, Molho ES, Kay DM, Montimurro J, et al. Postural instability/gait disturbance in Parkinson's disease has distinct subtypes: an exploratory analysis. J. Neurol. Neurosurg. Psychiatry 2011; 82: 564-8.

10. Vandenbossche J, Deroost N, Soetens E, Spildooren J, Vercruysse S, Nieuwboer A, et al. Freezing of Gait in Parkinson Disease Is Associated With Impaired Conflict Resolution Neurorehabil Neural Repair 2011; 25: 765-73.

11. Olmo MF, Arias P, Mazaira FJ. Facilitación de la actividad motora por estímulos sensoriales en la enfermedad de Parkinson. Revista Neurología 2004; 39: 841-7.

12. King LA, Horak FB. Delaying Mobility Disability in People with Parkinson Disease using a Sensorimotor Agility Exercise Program. Physical Therapy 2009; 89: 384-93.

13. Giladi N, Treves TA, Simon ES, Shabtai H, Orlov YB, Kandinov D, et al. Freezing of gait in patients with advanced Parkinson's disease. Journal of Neural Transmission 2001; 108: 53-61.

14. Rascol O, Brooks DJ, Korczyn AD, De Deyn PP, Clarke CE, Lang AE. A five-year study of the incidence of dyskinesia in patients with early Parkinson's disease who were treated with ropinirole or levodopa. The New England Journal of Medicine 2000; 342: 1484-91.

15. The Parkinson Study Group. Pramipexole vs Levodopa as Initial Treatment for Parkinson Disease. Arch Neurol 2004; 61: 1044-53.

16. Nicaretta DH, Pereira JS, Pimentel MLV. Primary progressive freezing gait - Case Report. Arq Neuropsiquiatr 1997; 55: 496-8.

17. Lebold CA, Almeida QJ. Evaluating the Contributions of Dynamic Flow to Freezing of Gait in Parkinson's Disease. Parkinson's Disease 2010; 2010: 1-7.

18. Olmo MFD, Cudeiro J. Temporal variability of gait in Parkinson disease: effects of a rehabilitation programme based on rhythmic sound cues. Parkinsonism and Related Disorders 2005; 11:25-33.

19. Nieuwboer A, Kwakkel G, Rochester L, Jones D, Van Wegen E, Willems AM, et al. Cue training in the home improves mobility in Parkinson's disease: the RESCUE trial. J Neurol Neurosurg Psychiatry 2007; 78: 134-40.

20. Aminian K, Najafi B, Bula C, Leyvraz PF, Robert P. Spatio-temporal parameters of gait measured by an ambulatory system using miniature gyroscopes. J Biomech 2002; 35: 689-99.

21. Salarian A, Russmann H, Vingerhoets FJ, Dehollain C, Blanc Y, Burkhard PR, et al. Gait assessment in Parkinson's disease: toward an ambulatory system for long-term monitoring. IEEE Trans Biomed Eng 2004; 51: 1434-43.

22. Van Wegen E, Lim I, de Goede C, Nieuwboer A, et al. The effects of visual rhythms and optic flow on stride patterns of patients with Parkinson's disease. Parkinsonism Relat Disord 2006; 12: 21-7.

23. Kompoliti K, Goetz CG, Leurgans S, Morrissey M, Siegel IM. On' freezing in Parkinson's disease: resistance to visual cue walking devices, Movement Disorder 2000; 15: 309-12.

24. Rochester L, Hetherington V, Jones D, Nieuwboer A, Willems AM, Kwakkel G, et al. The effect of external rhythmic cues (auditory and visual) on walking during a functional task in homes of people with Parkinson's disease. Arch Phys Med Rehabil 2005; 86: 999-1006.

25. Lo AC, Chang VC, Gianfrancesco MA, Friedman JH, Patterson TS, Benedicto DF. Reduction of freezing of gait in Parkinson's disease by repetitive robot-assisted treadmill training: a pilot study. Journal of Neuroeng and Rehabilitation 2010, 7: 51.

26. Snijders AH, Bloem BR. Cycling for Freezing of Gait. The new england journal of medicine 2010. 362: e46. Disponível em: www.nejm.org. [17.07.2011].

27. Espay AJ, Dwivedi AK, Payne M, Gaines L, Vaughan JE, Maddux BN, et al. Methylphenidate for gait impairment in Parkinson disease: A randomized clinical trial. Neurology 2011; 76: 1256-62.

28. Nashatizadeh MM, Jankovic J. Treatment of Freezing of Gait in Parkinson's Disease with Deep Brain Stimulation of the Pedunculopontine Nucleus, Annals of 60 th Annual Meeting, American Academy of Neurology, Chicago, USA, april 2008.

29. Thevathasan W, Pogosyan A, Hyam JA, Jenkinson N, Bogdanovic M, Coyne TJ, et al. A block to pre-prepared movement in gait freezing, relieved by pedunculopontine nucleus stimulation. Brain July 2011; 134: 2085-95.

30. Moreau C, Defebvre L, Deste'e A, Bleuse S, Clement F, Blatt JL, et al. STN-DBS frequency effects on freezing of gait in advanced Parkinson disease. Neurology 2008; 71: 80-4. 